\title{
Effect of isokinetic training on shoulder impingement
}

\author{
T.L. Wang ${ }^{1}$, B.M. Fu ${ }^{2}$, G. Ngai ${ }^{3}$ and P. Yung ${ }^{2,3}$ \\ ${ }^{1}$ Rehabilitation Department, DeltaWest Clinic, Delta Hospital, \\ Shanghai, China \\ ${ }^{2}$ Orthopedics Department, Prince of Wales Hospital, Hong Kong, China \\ ${ }^{3}$ Chinese University of Hong Kong, Shatin, Kowloon, Hong Kong, China \\ Corresponding authors: T.L. Wang / P. Yung \\ E-mail: taoliwangchina@yeah.net / patrickyung@126.com
}

Genet. Mol. Res. 13 (1): 744-757 (2014)

Received May 21, 2013

Accepted November 12, 2013

Published January 31, 2014

DOI http://dx.doi.org/10.4238/2014.January.31.1

\begin{abstract}
The aim of this study was to review the literature evaluating the effect of isokinetic training in patients suffering from shoulder impingement syndrome (SIS). Studies published up to March 2011 were located from the Pubmed, Scopus, Lilacs, Physiotherapy Evidence Database, and Cochrane Library databases using "isokinetic", "shoulder", and "impingement" as key words. Referenced studies were also checked. Studies were included if isokinetic training was employed as at least one of the treatments in the therapeutic program to treat shoulder impingement or other shoulder pathologies leading to impingement-related pain. All eligible studies described the level of evidence, patient characteristics, interventions, outcome evaluation, results, complications, and return to work. There were 2 randomized control trials (RCTs) and 4 studies with level 4 evidence that met the inclusion criteria. All of the studies included showed a statistically or clinically significant outcome after isokinetic training. However, most of the studies could not identify the isolated effect of isokinetic training. There was not enough evidence to support or refute the effectiveness of isokinetic training for SIS. This result does not reflect a true lack
\end{abstract}


of effect, but rather a lack of RCTs. A consensus definition of the different types and stages of SIS is urgently needed. More RCTs are also essential to clarify the value of this technique. The homogeneity of treatment interventions, study populations, and outcome measures should be prioritized. Further studies are also needed to clarify the differences in isokinetic data across different types and stages of shoulder impingement.

Key words: Isokinetic; Shoulder; Impingement

\section{INTRODUCTION}

Shoulder pain is second only to lower back pain in occurrence, affecting approximately 16 to $21 \%$ of the population (Michener et al., 2004). Impingement is one of the most common shoulder disorders, accounting for 44-65\% of all complaints of shoulder pain (Mattiello-Rosa et al., 2008). However, it is not easy to establish the accurate and isolated incidence of shoulder impingement. This is partly due to the fact that in the existing literature, impingement is generally described as a group of symptoms rather than a specific diagnosis, and is relatively difficult to define (Henrichs and Stone, 2004). In addition, impingement is strongly associated with other shoulder pathologies in both ordinary patients and symptomatic athletes. For instance, Warner et al. (1990) indicated that $68 \%$ of patients with instability had significant impingement signs in addition to apprehension and capsular laxity. Many adults older than 60 years of age have a partial or complete rotator cuff (RTC) tear, and many have impingement signs (Figure 1). As outlined by van der Heijden (1999) in his review of shoulder disorders, there is no gold standard that provides a valid and reliable estimate for clinically relevant changes in patients suffering from shoulder pathologies. Based on his review, the main outcome measures were suggested to be focused on the following elements: pain, range of motion, strength, and functional outcomes. Fortunately, conservative treatment is the first choice for most patients suffering from shoulder impingement, and the majority of these patients will recover with appropriate non-operative treatments (Henrichs and Stone, 2004). After a period of time of nonsurgical therapy, generally from 3 to 6 months, if the outcome is unsatisfactory, several surgical options should be considered (Henrichs and Stone, 2004). A systematic review concluded that strengthening of the RTC and scapular stabilizing muscles and stretching the soft tissues of the anterior and posterior shoulder were the options most supported by the current literature, and were recommended over no treatment or placebo treatment for subacromial impingement syndrome (SAIS; Michener et al., 2004). In addition, the isokinetic dynamometer plays an important role for testing and training of the shoulder complex (Ellenbecker and Davies, 2000; Codine et al., 2005). This kind of device can provide accommodating resistance to the movement of a patient. Therefore, no matter how much effort is exerted, the movement takes place at a constant preset speed, and the isokinetic exercise is relatively safe for patients (Codine et al., 2005). An increasing number of studies have indicated that the imbalance of the RTC is an important cause in the occurrence of impingement. This kind of imbalance can be expressed as changes in the strength ratio (peak torque ratio) of the cuff by isokinetics (Leroux et al., 1994). In addition, isokinetic training succeeded in enhancing the strength to redress the imbalance of shoulder muscle groups and the performance of normal subjects (Malliou et al., 2004). The emphasis is placed on the testing and training of the internal and external rotation strength of the shoulder. Davies (1984) and Malliou 
et al. (2004) concluded that isokinetic strengthening was the most effective method of altering the strength ratios of the RTC compared with isolated isotonic training, multi-joint dynamic resistance training, and no intervention. However, a qualitative review in 2000 (Ellenbecker and Davies) concluded that isokinetic treatment techniques were only one part of the rehabilitation process, which is tremendously diverse. Furthermore, with respect to shoulder impingement, the use of isokinetics as a treatment is relatively more rare and published less often compared to its use as an assessment tool (Codine et al., 2005). The purpose of the present review was to search the literature to identify the effect of isokinetic training in patients suffering from shoulder impingement syndrome (SIS).

\section{Shoulder Impingement Syndrome}

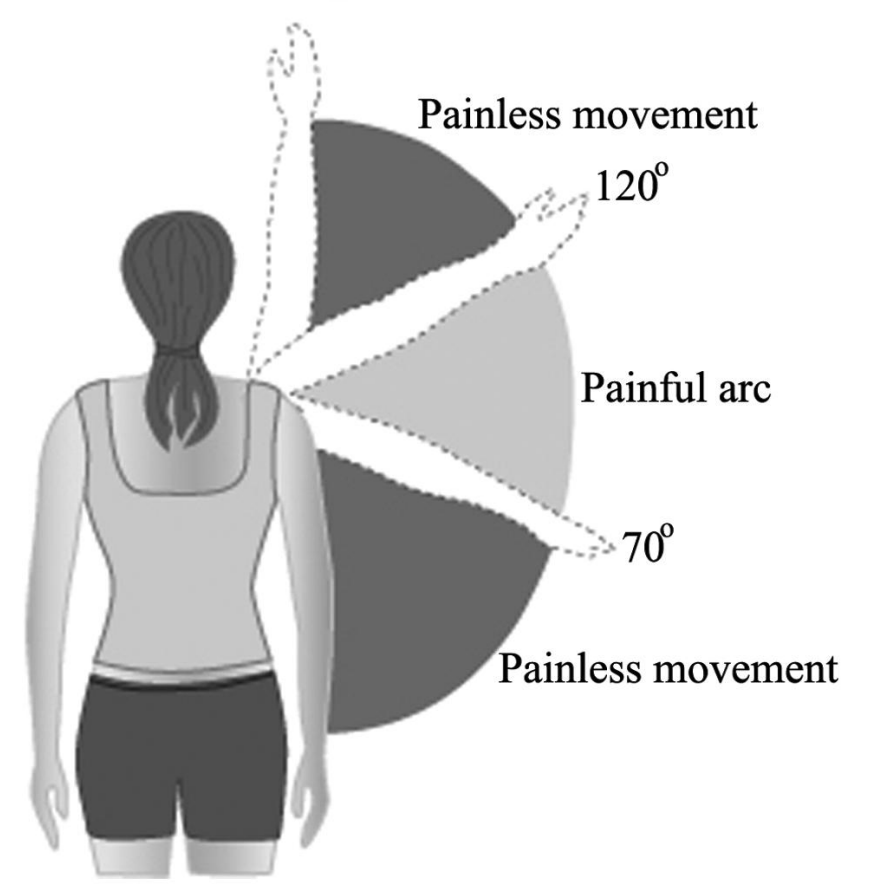

Figure 1. About $54 \%$ of adults older than 60 years have a partial or complete rotator cuff tear (Erol et al., 2008). Many of them have impingement signs.

\section{MATERIAL AND METHODS}

\section{Data source and literature search}

The computerized bibliographic databases Pubmed, Scopus, Lilacs, Physiotherapy Evidence Database, and the Cochrane Library were searched in sequence. The key words used were "isokinetic", "shoulder", and "impingement", with the Boolean operator "AND". All fields of the Scopus database were searched, and the Cochrane Library was searched by all 
text. There were no specific restrictions in the search fields of the other databases. No limits were included concerning the year of publication, and this search was conducted up to March 2011. No restrictions of language or study type were applied. In addition, the references of all studies retrieved were manually searched for further relevant studies.

\section{Study selection}

The inclusion criterion of this review was as follows: isokinetic training was employed as the main treatment or as at least one of the treatments of the whole therapeutic program in shoulder impingement or other shoulder pathologies with pain correlated to impingement. All titles and abstracts retrieved were read to assess their relevance for the first round of review. Any studies related to isokinetic training and impingement-related shoulder pain were selected for further examination of the full text for relevant studies or were otherwise excluded. The full text was also reviewed in any publications having unclear definitions of interventions used including physiotherapy, nonsurgical treatment, non-operative treatment, conservative treatment, specific muscular strengthening, post-operative programs, supervised exercise, comprehensive treatment, and so on. Similarly, the full text of studies was reviewed if they had an unclear definition of the diagnosis of the shoulder disorders involved. After the first round of review, the full text of the selected papers was assessed for inclusion in the second round of review. Additional articles were identified from the reference lists of relevant articles and were evaluated for inclusion according to the above criteria.

\section{Data extraction and quality assessment}

All eligible studies included descriptions of the level of evidence, patient characteristics, interventions, outcome evaluation, results, complications, and return to work. Descriptions of the information of the studies included are outlined in Table 1.

\begin{tabular}{llr}
\multicolumn{2}{c}{ Table 1. Yield from computerized search strategies. } & Number of articles \\
\hline Key words & Databases* & 35 \\
\hline Isokinetic & Pubmed & 505 \\
AND & Scopus (all fields) & 0 \\
Shoulder & Lilacs & 0 \\
AND & Physiotherapy Evidence Database & 5 \\
Impingement & Cochrane Library (all text) & 545 \\
& Total & 512 \\
\hline
\end{tabular}

*No limits were included concerning the year of publications, language and study type.

\section{RESULTS}

\section{Study selection}

In total, 512 articles (title and abstract) were retrieved from the electronic databases evaluated (Table 1). Five of these studies met the inclusion criteria. The flow path is presented in Figure 2. There were 4 studies extracted from 4 review articles (Hayes et al., 2002; Green et al., 2003; Codine et al., 2005; Seida et al., 2010), 2 of which were repeated. Among these 5 
eligible studies, there was only 1 randomized control trial (RCT; Reid et al., 1996) with level 2 evidence. Three studies were all uncontrolled pre- and post-test studies with level 4 evidence (Thomas et al., 2001; Manske and Davies, 2003; Boissonnault et al., 2007). Finally, there was one follow-up study that also had level 4 evidence (Martin and Garth, 1995). Two of the 5 studies used a compound diagnosis for patients (Thomas et al., 2001; Manske and Davies, 2003). These patients were diagnosed with a variety of shoulder pathologies, such as different types of impingement, shoulder instability, and RTC disorder, etc. The patients in the other 3 studies were diagnosed with sport-related isolated glenoid labral tears, symptomatic and atraumatic anterior instability, and type I and II RTC tears (Martin and Garth, 1995; Reid et al., 1996; Boissonnault et al., 2007). All patients had impingement-related shoulder pain. Two studies used isokinetic training as the main treatment method (Reid et al., 1996; Thomas et al., 2001). In contrast, the other 3 studies used isokinetic training as only a part of the whole therapeutic program (Martin and Garth, 1995; Manske and Davies, 2003; Boissonnault et al., 2007).

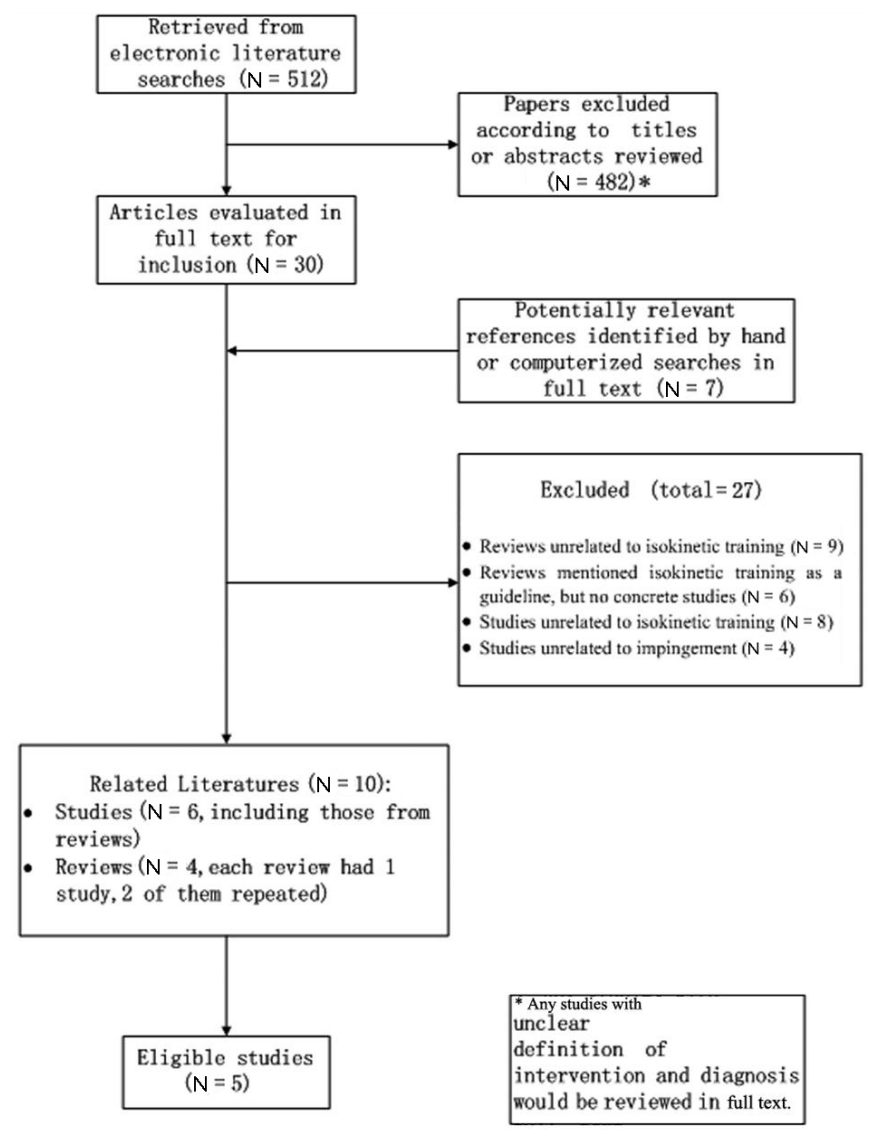

Figure 2. Literature search and selection.

There was a missing "relevant study" based on the current search strategy (McGee et al., 1999), which was found, coincidentally, by Dr. Davies, an expert in the field, using the search strategy of this review, and he kindly emailed it to us. It is unclear why this study was 
originally missed. This missing study is an RCT in which the patients were diagnosed with shoulder impingement, and Dr. Davies was one of the authors. Isokinetic training was integrated into the whole therapeutic program.

\section{Data extraction and quality assessment}

Further descriptions of the information of the eligible studies are as follows. In the study by Manske and Davies (2003), isokinetic training was integrated into the whole shoulder rehabilitation exercises in 2 intermediate phases. One was total arm strength, and the other was kinesthesia. In Martin and Garth (1995), isokinetic training was also a part of the whole post-operative rehabilitation program, in which the first stage was isotonic training. Once handmeasured external rotation (ER) and abduction strength progressed to the $5 / 5$ level, isokinetic concentric and eccentric ER training was initiated. In Boissonnault et al. (2007), a combined rehabilitation program was applied and included the isokinetic internal rotation (IR)/ER exercise in the supine or standing position 8 weeks after surgery. In addition, a standardized rehabilitation program was used in the study by Thomas et al. (2001), in which the major aspect was a special isokinetic pulley system. In an RCT conducted by Reid et al. (1996), 2 program groups were compared. The first program comprised of an isokinetic resistance exercise that was designed to improve muscle strength and endurance, and the other program consisted of electromyographic biofeedback re-education, which was designed to improve motor control. These 2 treatment groups were then compared with respect to function and strength at 8,26, and 52 weeks after program initiation. In addition, isokinetic training was integrated in the late stage of the so-called standard treatment protocol adopted in the missing study by McGee et al. (1999).

Isokinetic strength was the outcome evaluation of 4 studies (Martin and Garth, 1995; Reid et al., 1996; McGee et al., 1999; Thomas et al., 2001). There were also 4 studies that used function improvement as an outcome evaluation (Martin and Garth, 1995; Reid et al., 1996; McGee et al., 1999; Boissonnault et al., 2007). Pain assessment was another outcome evaluation metric that was used in 3 studies (Martin and Garth, 1995; Reid et al., 1996; McGee et al., 1999). Boissonnault et al. (2007) took the patient's psychological condition into consideration when assessing the impact of medical comorbidities selected on rehabilitation outcomes. One study (Manske and Davies, 2003) used torque-acceleration-energy (TAE) and TAE percentage deficits as the outcome evaluation metrics. TAE, one parameter of isokinetic dynamometry, is defined as the greatest amount of work performed in the first $125 \mathrm{~ms}$ of torque production in a singletest repetition. TAE measures the muscle's time rate of torque development, or neuromuscular stability, and describes the muscle's ability to generate torque quickly, which might be of more importance in athletics from a functional-performance standpoint (Manske and Davies, 2003).

The three studies using isokinetic strength for outcome evaluation showed statistically significant increases (Reid et al., 1996; McGee et al., 1999; Thomas et al., 2001). Another follow-up study revealed a $4.4 \%$ increase in concentric external rotation strength with an average of $90^{\circ}$ and an increase of $8.6 \%$ with an average of $180 \%$ s in the injured arm as compared with the opposite uninjured limb among 17 patients who underwent final isokinetic strength assessment (Martin and Garth, 1995). In the case of function outcome, statistically significant improvement was shown after isokinetic training in 1 pre- and post-test study (Boissonnault et al., 2007). The other 2 studies did not show statistically significant improvements, but did show clinically significant improvements in function (Reid et al., 1996; McGee et al., 1999). McGee et al. (1999) indicated that pain was decreased, but the data were not statistically analyzed due to the small 
sample size. Martin and Garth (1995) found that 5 of 9 patients with pre-operatively positive impingement signs achieved excellent results in their follow-up study. In another study (Manske and Davies, 2003), a statistically significant improvement was found in the outcome of TAE.

There were some additional discoveries made in the course of our review. Previous authors have established that the concentric isokinetic IR/ER strength ratio (peak torque ratio) in the normal population ranges from 1.3 to 1.6 (Davies, 1984). The concentric isokinetic IR/ER strength ratio was found to increase and tended toward 2 in the impingement population (Warner et al., 1990; Rupp et al., 1995; Rokito, 1996; Forthomme et al., 2003; MacDermid et al., 2004; Tyler et al., 2005). However, some other authors found the opposite outcome, showing that the isokinetic IR/ER strength ratio decreased and tended toward 1 in impingement patients (Rokito et al., 1996; Bak and Magnusson, 1997; Erol et al., 2008). In addition, Davies and DickoffHoffman (1993) found a nearly 1:1 relationship of concentric protraction/retraction isokinetic strength ratio in 250 shoulders. Cools et al. (2005) found a variety of alterations in the concentric scapular muscle isokinetic strength in overhead athletes with shoulder impingement symptoms. These changes included a statistically significant lower protraction/retraction strength ratio, indicating a weakened serratus anterior muscle and decreased and delayed muscle activities in the lower trapezius in shoulder movement, when isokinetics was combined with electromyography.

\section{Data synthesis and analysis}

Controlled studies and their results can be combined using meta-analysis if the study design, study population, interventions being compared, and outcomes are sufficiently similar. The Revman 5.0 software would be used to conduct the meta-analysis, and statistical heterogeneity would be quantified by using the $\mathrm{I}^{2}$ statistic.

Four of the 6 studies included were uncontrolled studies (Martin and Garth, 1995; Thomas et al., 2001; Manske and Davies, 2003; Boissonnault et al., 2007), and the other 2 studies were RCTs. However, one of the RCTs, which was emailed to us by Dr. Davies, was designed to identify the effect of closed kinetic chain exercise (McGee et al., 1999), and both groups in this study received isokinetic training as a part of the treatment of the whole therapeutic program; therefore, this study could not identify the isolated effect of isokinetic training. Ultimately, there was only 1 RCT identifying the effect of isokinetic training on impingement-related shoulder pathologies (Reid et al., 1996).

Furthermore, there was a lack of homogeneity among these studies with respect to study population, intervention, and outcome evaluation. Therefore, pooling of the data for a metaanalysis was not possible.

\section{DISCUSSION}

To achieve the objective of the present review, one problem had to be addressed in advance: how to define impingement as a clinical syndrome. SIS was first described by Jarjavay in 1867, but it was Neer who first popularized the term "impingement" in 1972. However, significant advancement in understanding of the anatomy and biomechanics of the human shoulder in the past 30 years has contributed to the identification of various types of impingement (Ludewig and Braman, 2011). The clinical presentations of SIS depend on the location of the impingement (Henrichs and Stone, 2004). By location, the shoulder impingement can be divided into 3 groups: external impingement or SAIS, internal or undersurface impingement, and coracoid 
impingement (Henrichs and Stone, 2004; Ludewig and Braman, 2011). External impingement or SAIS was first termed by Neer (Ludewig and Braman, 2011). External impingement occurs in the space between the inferior acromial and superior surface of the RTC tendons. Subsequently, Neer further divided external impingement into outlet and non-outlet lesions. Outlet impingement occurs when the coracoacromial arch infringes upon the supraspinatus outlet, whereas non-outlet impingement takes place secondary to thickening or hypertrophy of the bursa and/ or RTC tendons. In 1992, Walch et al. and Jobe CM et al. (unpublished results) independently described a novel impingement of the undersurface (deep layer) of the posterior supraspinatus tendon and/or the anterior infraspinatus tendon by the posterosuperior glenoid rim (Figures 3 and 4). This phenomenon became known as internal impingement, or posterior glenoid impingement. However, some authors have found that articular surface contact of the cuff with the glenoid labral complex can occur anteriorly/superiorly as well. Internal impingement was therefore divided into anterior or posterior impingement (Edelson and Teitz, 2000). Relative to external impingement, internal impingement typically affects young to middle-aged adults, and in most series of internal impingement, patients are under 40 years of age. A large majority of internal impingement patients are athletes (Figure 5). On the other hand, coracoid impingement refers to impingement of the subscapularis and/or adjacent soft tissues between the coracoid process and the lesser tuberosity. Although coracoid impingements are rare, it is important to differentiate them from other types of SIS since they usually require surgical treatment (Okoro et al., 2009).
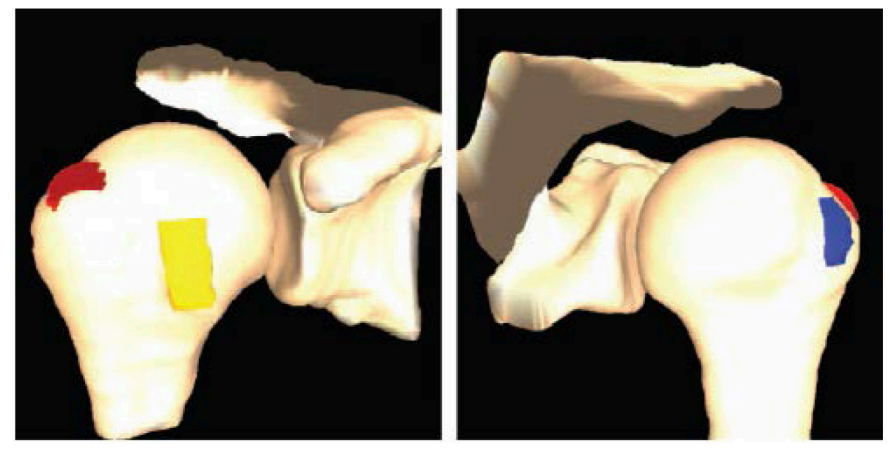

Figure 3. Neutral position of shoulder where blots represent subscapularis, supraspinatus, infraspinatus [it was extracted from the paper published by Gold et al. (2007)].
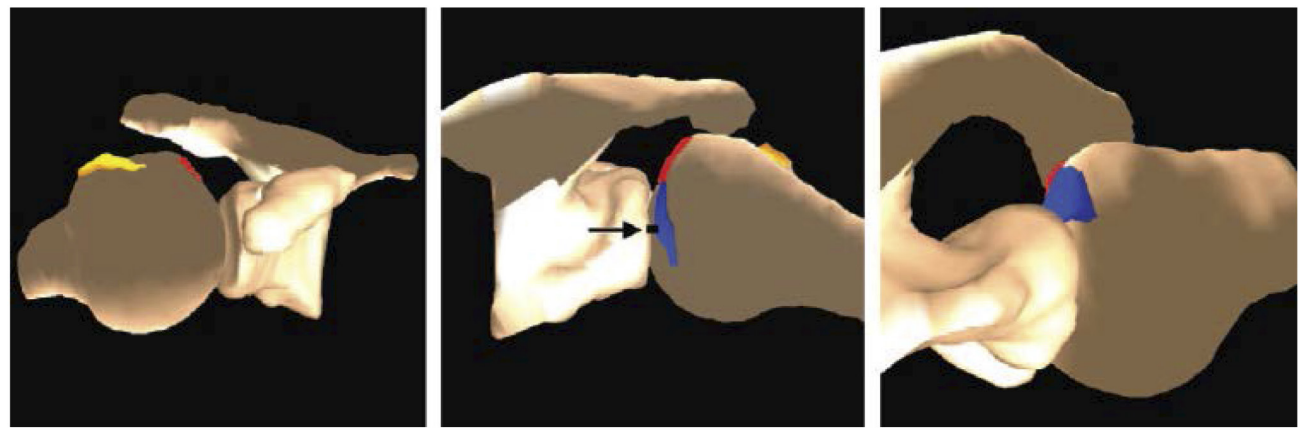

Figure 4. Maximal external rotation with $90^{\circ}$ abduction of shoulder. Contact occurs between glenoid rim and blots which represent supraspinatus and infraspinatus [is was extracted from the paper published by Gold et al. (2007)]. 


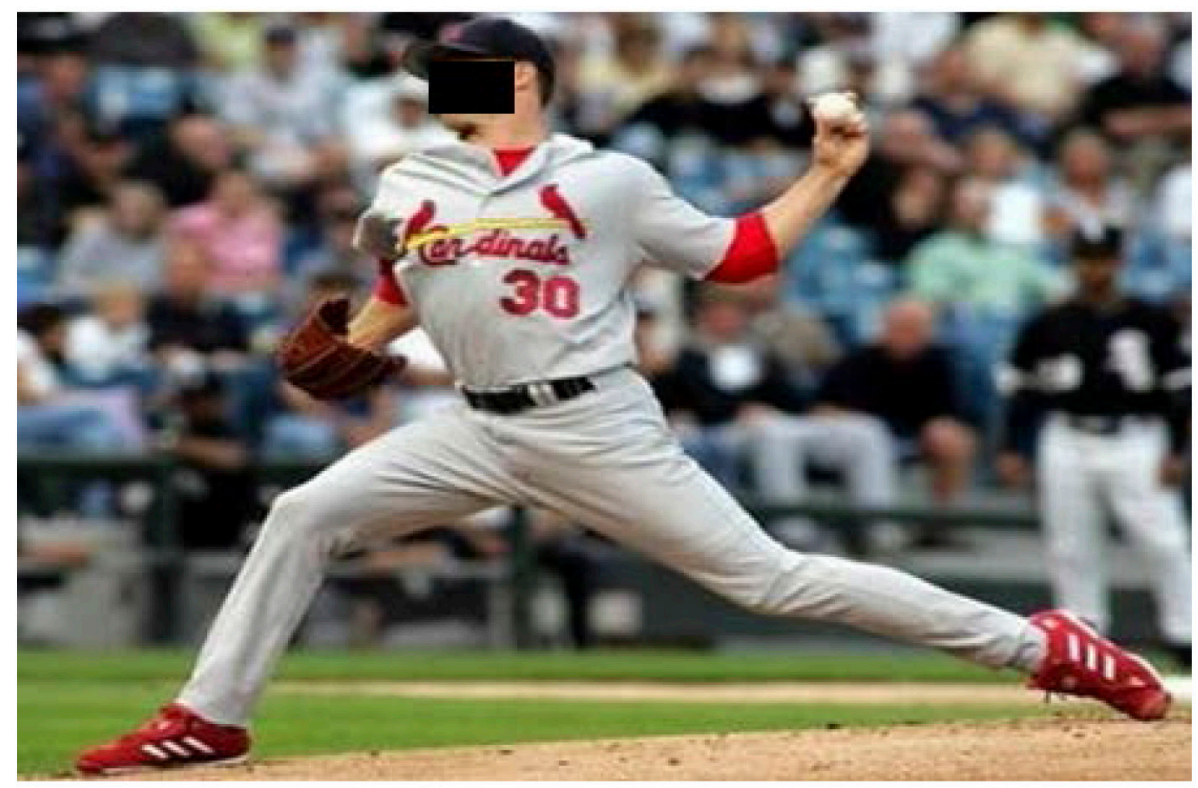

Figure 5. Relative to external impingement, internal impingement typically affects young to middle-aged adults, especially athlete patients.

The types of SIS described above are classified based on the location of the impingement. From another perspective, by its nature, SIS can be divided into primary or secondary shoulder impingement. Primary impingement mainly results from direct compression in the subacromial space or hypomobility, whereas secondary impingement is chiefly attributed to instability or hypermobility or to neurological injury.

Although several theories have been proposed about the etiology of shoulder impingements, it is believed that the main causes of impingement are multifactorial (Ludewig and Braman, 2011). There are 2 predominant theories about the causes of impingement: intrinsic and extrinsic. The intrinsic theory (intratendinous) supposes that degeneration of the tendons, attributed to overuse, tension overload, or trauma, can lead to muscle weakness with the subsequent lack of synergism of shoulder movement. The extrinsic theory (extratendinous) asserts that impingement occurs due to factors external to the tendon. These include faulty posture, acromial morphology, posterior capsular tightness, instability, and altered scapular or glenohumeral kinematics (Mattiello-Rosa et al., 2008).

Although shoulder pain is the most common musculoskeletal complaint faced in clinical settings after lower back pain, the clinical definition of the different problems characterized by shoulder pain remains controversial. To date, there is still noconsensus definition of SIS. It is worth noting that shoulder impingement often goes hand in hand with other shoulder pathologies. In addition, SIS is currently divided into 3 groups. In particular, SAIS, one of the most commonly concepts used to describe a category of shoulder problems, is classified as one type of SIS. Therefore, for this review, it was appropriate to retain studies dealing with any of the impingement-related diagnoses (e.g., functional shoulder instability, RTC disease, etc.). 
All of the studies included showed statistically or clinically significant improvement in outcome evaluation after isokinetic training. However, a majority of the studies had low evidence. Furthermore, most of these studies only used isokinetic training as part of the whole therapeutic program, and could not identify the isolated effects of isokinetic training on outcome. Therefore, these studies may have inaccurately estimated the effect of isokinetic training of all treatment effects. One RCT with only 20 participants (Reid et al., 1996) showed no statistically significant difference between a routine of isokinetic resistance exercises and the use of electromyographic biofeedback. Furthermore, the study could not provide any information about the benefit of exercise over lack of treatment. The other RCT (McGee et al., 1999) also failed to provide any evidence of isokinetic training. Therefore, there was not enough evidence to support or refute the effectiveness of isokinetic training for SIS. This does not reflect a true lack of effect, but a lack of RCTs in this area. Additionally, the homogeneity of eligible studies appeared to be poor. For instance, the different studies used various population types (athletes or not, different age groups, etc.), different types and stages of shoulder impingement, different training positions, different sample sizes, and different testing pain-free range of motion (ROM), which can all affect the comparison among studies (Leroux et al., 1994; Erol et al., 2008). These factors could partly explain why there were contrasting results in the testing of isokinetic IR/ER strength ratio of impingement patients, as mentioned above (Warner et al., 1990; Leroux et al., 1994; Rupp et al., 1995; Rokito, 1996; Bak and Magnusson, 1997; Forthomme et al., 2003; MacDermid et al., 2004; Tyler et al., 2005; Erol et al., 2008).

Previous studies have demonstrated a significant difference in the isokinetic strength of IR and ER, with varying degrees of abduction, flexion, and horizontal abduction and adduction of the glenohumeral joint. Isokinetic training is generally considered to have training specificity, although this remains controversial (Ellenbecker and Davies, 2000). It is therefore essential, as advocated by Codine et al. (2005), to establish a consensus on the testing and training position. A relatively ideal position, the so-called "modified base position", is increasingly being adopted (Figure 6) (Warner et al., 1990; Leroux et al., 1994). This position was first presented by Dr. Davies in the early 1980s, and has also been termed the " $30^{\circ} / 30^{\circ} / 30^{\circ}$ position" (Davies, 1984). The modified base position is obtained by tilting the dynamometer approximately $30^{\circ}$ from the horizontal base position. The patients' glenohumeral joint is placed at $30^{\circ}$ of abduction and $30^{\circ}$ of forward flexion into the plane of the scapula, with a $30^{\circ}$ diagonal tilt of the dynamometer head from the transverse plane. This position has many advantages. First, certain abductional positions of the shoulder, such as $30^{\circ} \mathrm{AB}$, can prevent a "wringing out" effect and increase blood flow (i.e., $\mathrm{O}_{2}$ and nutrients) to the RTC tendon. Second, the scapular plane can decrease the stress on the anterior shoulder capsule and ligaments so as to protect anterior structures, and can pre-stretch the posterior muscles to provide a better length-tension relationship of the scapulohumeral musculature. Third, the diagonal tilt plane places the RTC oblique muscle fibers (subscapularis, infraspinatus, teres minor) in direct line for force production, according to anatomy and biomechanics, to improve the dynamic caudal glide of the humeral head (Figure 7). Relative adduction activity can widen the subacromial space and protect its tissues, and it can also minimize deltoid activity compared with the "can" position. This position is comfortable for patients and has less of a chance of inducing impingement signs, which was an empirically based comment (Davies, 1984). 


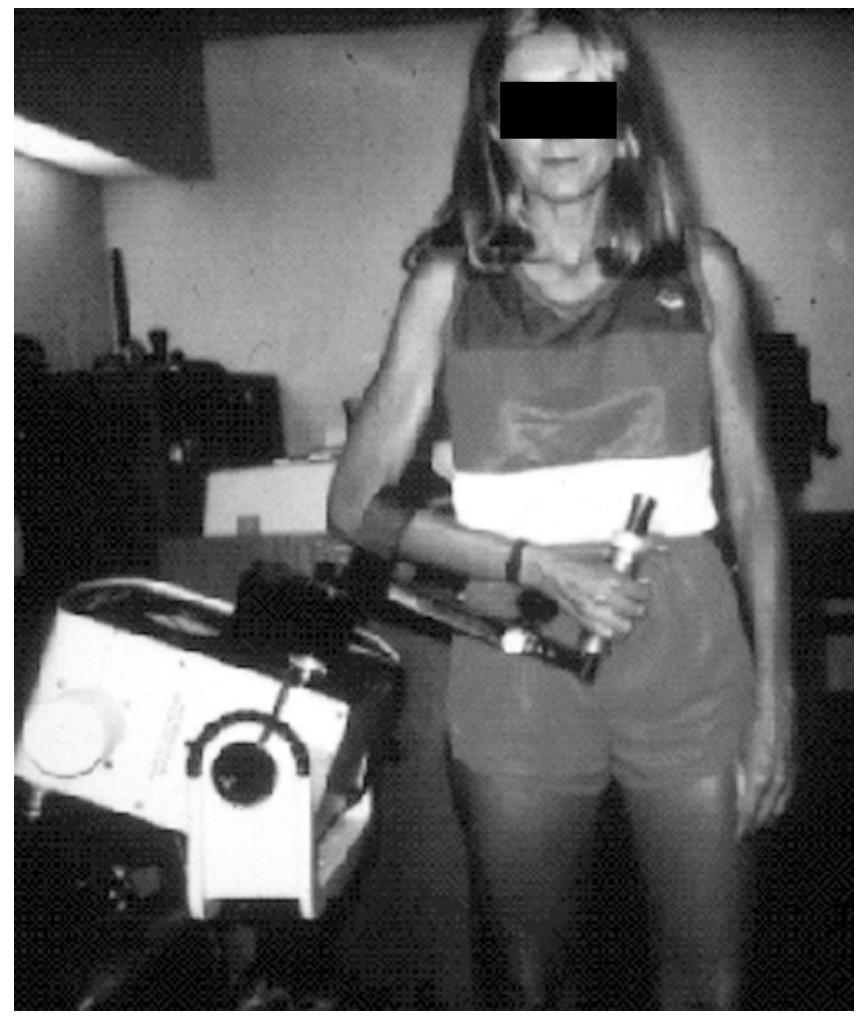

Figure 6. Modified base position $\left(30^{\circ} / 30^{\circ} / 30^{\circ}\right)$.

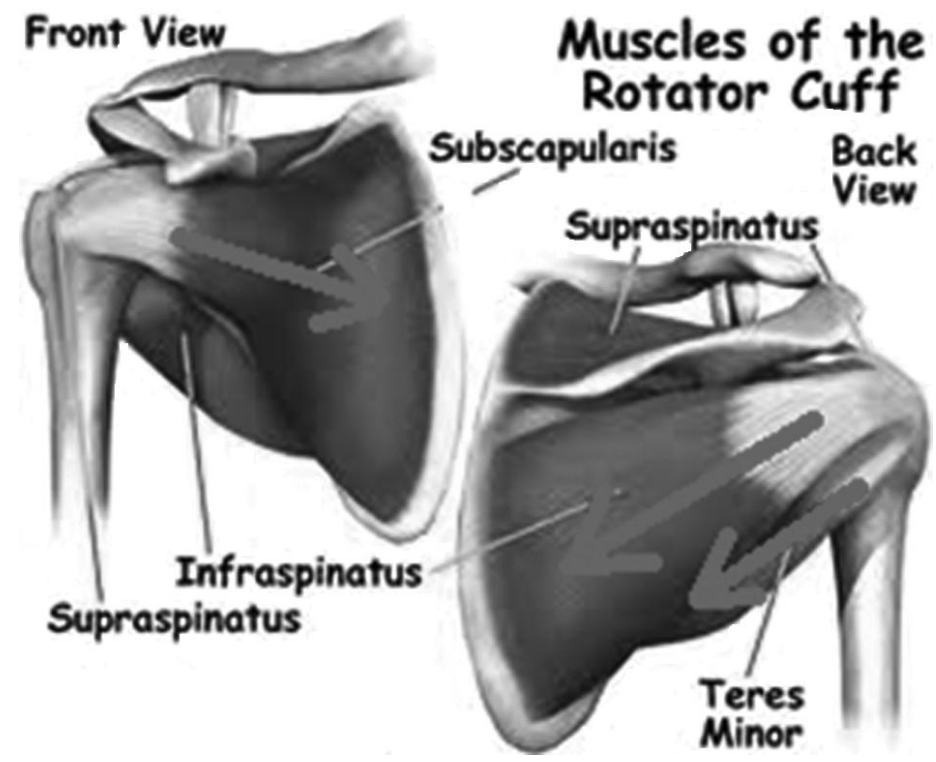

Figure 7. Oblique fiber orientation creates the dynamic caudal glide. 
Different types and stages of shoulder impingement appear to have diverse peak torque ratio changes by isokinetic device (Leroux et al., 1994; Erol et al., 2008). It is understandable that different mechanisms of anatomy and biomechanics can lead to different outcomes of pathologies. The natural history of shoulder impingement was postulated by Neer's original paper, which was actually based on the nature of SAIS, one type of SIS. Neer's stage I was classified by edema and hemorrhage of the subacromial bursa and RTC muscles. These findings were typically found in patients younger than 25 years. In stage II, fibrosis and tendinosis were apparent, which were irreversible. These patients were typically between 25 and 40 years old. In stage III, subacromial impingement, or external impingement, was marked by partial and complete tearing of the RTC, and was generally found in patients older than 40 years (Henrichs and Stone, 2004). Hawkins and Kennedy (1980) agreed with the concept of Neer's 3 stages, but strongly suggested that impingement finally affected the biceps tendon, subacromial bursa, and the acromioclavicular joint. They also thought that the onset of impingement could occur at virtually any age. Therefore, they proposed a different version of the 3 stages (Hawkins and Kennedy, 1980). In stage I, patients had very variable presentations, with either a tendinitis and limitation in ROM, or minimal pain with activity and no weakness. Either the biceps tendon or the supraspinatus tendon could be involved, so that patients could present a painful arc of abduction or tenderness over the biceps tendon, with tests for biceps tendinitis producing pain. In stage II, repeated insults to the supraspinatus tendon, biceps tendon, and subacromial bursa led to progression of symptoms. Patients described a stiffer shoulder, and greater inability to perform the offending movement. Rest frequently did not resolve symptoms, as it often did in stage I. In stage III, patients described toothache-like pain, which limited athletic activity, and usually RTC tears, especially after the age of 50 years and was seldom seen under the age of 40 years. These tearings often presented sudden weakness and diminished ROM. There were also more frequent radiograph findings in stage III, with osteophytes, sclerosis, and cystic degeneration of the greater tuberosity (Hawkins and Kennedy, 1980). However, with 30 years of development in basic scientific research in the areas of anatomy and biomechanics of the human shoulder, some authors have more recently presented a modified schema of SAIS in which the 3 stages are considered to be RTC tendonitis with no tearing, partial RTC tearing, and full thickness tears of the RTC (Park et al., 2004). On the other hand, with respect to shoulder internal impingement, Jobe (1996) described 3 stages that could help identify patients before the onset of shoulder pain. In stage I, the athlete complains of stiffness and needs more time to warm up. It is at this point that early recognition and appropriate rehabilitation may allow the athlete to return to play in a short period. Stage II occurs when the patient develops pain and a positive relocation test. Stage III is the same as stage II, but with the failure of a rehabilitation program. The discrepancy of the isokinetic strength ratio, which might exist in different populations studied, might result, at least in part, from different types and/or stages of shoulder impingement. To date, however, there has been a lack of uniformity in defining, evaluating, and treating SIS, which has impeded further clinical and research study. Therefore, it is very important to specify the type and stage when diagnosing and treating a patient suffering from shoulder impingement.

Almost all of the shoulder impingement patients were tested and treated by isokinetics in their pain-free ROM of shoulder joint. It should be pointed out that different testing and training ROM might result in different outcomes. Because this issue was not the main objective of the current review, this problem will not be addressed further here. 
An increasing number of studies have highlighted the importance of scapular muscles in the onset of shoulder impingement (Cools et al., 2005). It is commonly necessary to perform isolated testing and training of specific muscle groups affected by certain pathological changes as every weak component part of the whole kinetic chain should be identified and adequately rehabilitated (Ellenbecker and Davies, 2000). The whole kinetic chain is always only as strong as its weakest link, increasing proximal stability, and enhancing distal mobility (Davies, 1984).

There is currently not enough evidence available to support or refute the effectiveness of isokinetic training for SIS. This does not reflect a true lack of effect, but rather a lack of RCTs in this area. A consensus definition of the different types and stages of SIS is urgently needed to promote further clinical and research study. It is also essential to conduct studies providing a sufficient level of evidence (i.e., RCTs), with and without isokinetic training, and with large sample sizes in order to identify the true value of this technique. In addition, the homogeneity of treatment interventions, study populations, and outcome measures should be prioritized. The position of isokinetic training and testing on the shoulder also needs to be standardized to improve comparability and research. The modified base position $\left(30^{\circ} / 30^{\circ} / 30^{\circ}\right)$ is one good choice. Further studies are also needed to clarify the differences in abnormal isokinetic data among different types and stages of shoulder impingement. Again, an adequate sample size for such studies is essential. Finally, more attention should be paid to the application of isokinetic training and testing on scapular muscle groups in shoulder impingement patients.

\section{REFERENCES}

Bak K and Magnusson SP (1997). Shoulder strength and range of motion in symptomatic and pain-free elite swimmers. Am. J. Sports Med. 25: 454-459.

Boissonnault WG, Badke MB, Wooden MJ, Ekedahl S, et al. (2007). Patient outcome following rehabilitation for rotator cuff repair surgery: the impact of selected medical comorbidities. J. Orthop. Sports Phys. Ther. 37: 312-319.

Codine P, Bernard PL, Pocholle M and Herisson C (2005). Isokinetic strength measurement and training of the shoulder: methodology and results. Ann. Readapt. Med. Phys. 48: 80-92.

Cools AM, Witvrouw EE, Mahieu NN and Danneels LA (2005). Isokinetic Scapular Muscle Performance in Overhead Athletes With and Without Impingement Symptoms. J. Athl. Train. 40: 104-110.

Davies GJ (1984). A Compendium of Isokinetics in Clinical Usage and Rehabilitation Techniques. 1st edn. WI: S \& S Publishers, LaCrosse.

Davies GJ and Dickoff-Hoffman S (1993). Neuromuscular testing and rehabilitation of the shoulder complex. J. Orthop. Sports Phys. Ther. 18: 449-458.

Edelson G and Teitz C (2000). Internal impingement in the shoulder. J. Shoulder Elbow Surg. 9: 308-315.

Ellenbecker TS and Davies GJ (2000). The application of isokinetics in testing and rehabilitation of the shoulder complex. J. Athl. Train. 35: 338-350.

Erol O, Ozcakar L and Celiker R (2008). Shoulder rotator strength in patients with stage I-II subacromial impingement: relationship to pain, disability, and quality of life. J. Shoulder Elbow Surg. 17: 893-897.

Forthomme B, Croisier JL, Huskin JP and Crielaard JM (2003). Isokinetic assessment of shoulders with impingement syndrome following pain inhibition. Isokinetics Exerc. Sci. 11: 70-71.

Gold GE, Pappas GP, Blemker SS, Whalen ST, et al. (2007). Abduction and external rotation in shoulder impingement: an open MR study on healthy volunteers - initial experience. Radiology 244: 815-822.

Green S, Buchbinder R and Hetrick S (2003). Physiotherapy interventions for shoulder pain. Cochrane Database Syst. Rev. CD004258.

Hawkins RJ and Kennedy JC (1980). Impingement syndrome in athletes. Am. J. Sports Med. 8: 151-158.

Hayes K, Callanan M, Walton J, Paxinos A, et al. (2002). Shoulder instability: management and rehabilitation. J. Orthop. Sports Phys. Ther. 32: 497-509.

Henrichs J and Stone D (2004). Shoulder impingement syndrome. Prim. Care 31: 789-805, vii.

Jobe CM (1996). Superior glenoid impingement. Current concepts. Clin. Orthop. Relat. Res. 98-107. 
Leroux JL, Codine P, Thomas E, Pocholle M, et al. (1994). Isokinetic evaluation of rotational strength in normal shoulders and shoulders with impingement syndrome. Clin. Orthop. Relat. Res. 108-115.

Ludewig PM and Braman JP (2011). Shoulder impingement: biomechanical considerations in rehabilitation. Man. Ther. 16: 33-39.

MacDermid JC, Ramos J, Drosdowech D, Faber K, et al. (2004). The impact of rotator cuff pathology on isometric and isokinetic strength, function, and quality of life. J. Shoulder Elbow Surg. 13: 593-598.

Malliou PC, Giannakopoulos K, Beneka AG, Gioftsidou A, et al. (2004). Effective ways of restoring muscular imbalances of the rotator cuff muscle group: a comparative study of various training methods. Br. J. Sports Med. 38: 766-772.

Manske RC and Davies GJ (2003). Postrehabilitation outcomes of muscle power (torque-acceleration energy) in patients with selected shoulder dysfunctions. Acta Orthop. Scandinavica 74: 408-414.

Martin DR and Garth WP Jr (1995). Results of arthroscopic debridement of glenoid labral tears. Am. J. Sports Med. 23: 447-451.

Mattiello-Rosa SM, Camargo PR, Santos AA, Pádua M, et al. (2008). Abnormal isokinetic time-to-peak torque of the medial rotators of the shoulder in subjects with impingement syndrome. J. Shoulder Elbow Surg. 17: 54S-60S.

McGee C, Kersting E, Palmer-McLean K and Davies GJ (1999). Standard rehabilitation vs standard plus closed kinetic chain rehabilitation for patients with shoulder impingement: a rehabilitation outcomes study. Phys. Ther. 79: s65.

Michener LA, Walsworth MK and Burnet EN (2004). Effectiveness of rehabilitation for patients with subacromial impingement syndrome: a systematic review. J. Hand Ther. 17: 152-164.

Okoro T, Reddy VR and Pimpelnarkar A (2009). Coracoid impingement syndrome: a literature review. Curr. Rev. Musculoskelet. Med. 2: 51-55.

Park HB, Lin SK, Yokota A and McFarland EG (2004). Return to play for rotator cuff injuries and superior labrum anterior posterior (SLAP) lesions. Clin. Sports Med. 23: 321-34, vii.

Reid DC, Saboe LA and Chepeha JC (1996). Anterior shoulder instability in athletes: comparison of isokinetic resistance exercise and an electromyographic biofeedback re-education program-a pilot program. Physiother. Can. 48: 251-256.

Rokito AS, Zuckerman JD, Gallagher MA and Cuomo F (1996). Strength after surgical repair of the rotator cuff. $J$. Shoulder Elbow Surg. 5: 12-17.

Rupp S, Berninger K and Hopf T (1995). Shoulder problems in high level swimmers - impingement, anterior instability, muscular imbalance? Int. J. Sports Med. 16: 557-562.

Seida JC, LeBlanc C, Schouten JR, Mousavi SS, et al. (2010). Systematic review: nonoperative and operative treatments for rotator cuff tears. Ann. Intern. Med. 153: 246-255.

Thomas M, Grunert J, Standtke S and Busse MW (2001). Rope pulley isokinetic system in shoulder rehabilitation - initial results. Z. Orthop. Ihre Grenzgeb 139: 80-86.

Tyler TF, Nahow RC, Nicholas SJ and McHugh MP (2005). Quantifying shoulder rotation weakness in patients with shoulder impingement. J. Shoulder Elbow Surg. 14: 570-574.

van der Heijden GJ (1999). Shoulder disorders: a state-of-the-art review. Baillieres Clin. Rheumatol. 13: 287-309.

Walch G, Boileau P, Noel E and Donell ST (1992). Impingement of the deep surface of the supraspinatus tendon on the posterosuperior glenoid rim: an arthroscopic study. J. Shoulder Elbow Surg. 1: 238-245.

Warner JJ, Micheli LJ, Arslanian LE, Kennedy J, et al. (1990). Patterns of flexibility, laxity, and strength in normal shoulders and shoulders with instability and impingement. Am. J. Sports Med. 18: 366-375. 